\title{
Polymer Embolism from Bioactive and Hydrogel Coil Embolization Technology: Considerations for Product Development
}

M odified (bioactive and hydrogel) coil embolization technologies were introduced to reduce aneurysm recurrence rates. The addition of bioactive polymers to bare platinum coils is designed to induce a tissue response and promote neoendothelialization. Hydrogel polymers on platinum coils swell in contact with the physiologic environment, filling the aneurysmal sac, and possibly increasing occlusion rates.

Reinges et $\mathrm{al}^{1}$ animal study reported on rates of vessel wall reconstruction over the aneurysm neck with modified coil technologies. After 12 months, the study determined a minimal neointimal or neoendothelial layer for bioactive coils and complete aneurysm obliteration and neoendothelialization for hydrogel coils at the aneurysm neck. For aneurysms treated with hybrid bare platinum-hydrogel coils, a postmortem study of 13 human aneurysms retrieved between 1 and 74 days postembolization reported that neointima formation had begun at 5 days and a new vessel wall supported by smooth-muscle cells (at the margins) had traversed the aneurysm neck at 74 days. $^{2}$

Notably, until the clot-coil complex is formed and the aneurysm neck is bridged, bioactive and hydrogel polymers on platinum coils are exposed to continuous pulsatile blood flow. Polymers on intravascular devices exposed to aqueous environments are known to avulse in a relatively short time. For example, an in vitro test highlights shedding of polymer from the distal end of a guide catheter immersed in $0.9 \%$ saline in as little as 60 minutes. ${ }^{3}$ Of relevance, the average time it takes for the aforementioned physiologic processes to occur (a few days) far exceeds the time it takes for polymers to detach from the modified coils when exposed to an aqueous environment. For embolization coils, polymer separation begins when coil loops form and interact with each other on deployment within the aneurysmal sac, resulting in shedding and possible displacement of the polymer material. Stent-assisted coiling and coil-supported flow diversion may further facilitate mechanical abrasion and subsequent polymer detachment. Polymer embolism from coil embolization procedures has been associated with the formation of round enhancing lesions and surrounding vasogenic edema, resulting in neurologic decline between 1 and 270 days from the coiling procedure (average, 1-2

http://dx.doi.org/10.3174/ajnr.A6083 months). ${ }^{3}$ In reported events, even though the edema was effectively treated with corticosteroids, some patients were dependent on medications with remnant brain lesions years after the initial procedure. ${ }^{3}$

The 2015 US FDA safety communication sheds light on this iatrogenic complication of polymer embolism and states that it will work with stakeholders to close gaps in standards related to polymer coating integrity. ${ }^{4}$ Currently particulate testingdetermining the count and size of polymer particulates separated from a device when tested in a clinically representative environment - is the standard for evaluating coating integrity on an intravascular device. ${ }^{5}$ Given the ubiquitous use of hydrophilic coatings on neurovascular devices, mitigating particulates may be the preferred method of addressing this complication, especially from permanently implantable devices. Improvements in coil embolization technology with the addition of bioactive and hydrogel polymers emphasize the importance of particulate testing to determine, mitigate, and possibly eliminate the impact of this potential iatrogenic complication from these important neurovascular technologies. This information is relevant to physicians, regulators, design engineers, and manufacturers for product development as newer generations of embolization coils become available for use.

Disclosures: Juan Pablo Cruz-UNRELATED: Payment for lectures including service on speakers bureaus: Roche, Comments: a talk regarding the role of MR imaging in the pharmacovigilance for progressive multifocal leukoencephalopathy (PML) in MS for a national congress.

\section{REFERENCES}

1. Reinges MH, Krings T, Drexler AY, et al. Bare, bio-active and hydrogel-coated coils for endovascular treatment of experimentally induced aneurysms: long-term histological and scanning electron microscopy results. Interv Neuroradiol 2010;16:139-50 CrossRef Medline

2. Killer M, Arthur AS, Barr JD, et al. Histomorphology of thrombus organization, neointima formation, and foreign body response in retrieved human aneurysms treated with hydrocoil devices. J Biomed Mater Res B Appl Biomater 2010;94:486-92 CrossRef Medline

3. Chopra AM, Mehta M, Bismuth J, et al. Polymer coating embolism from intravascular medical devices: a clinical literature review. Cardiovasc Pathol 2017;30:45-54 CrossRef Medline

4. U.S. Food and Drug Administration Safety Communications. Lubricious Coating Separation from Intravascular Medical Devices: 
FDA Safety Communication. November 23, 2015. http://wayback. archive-it.org/7993/20170404182155/https://www.fda.gov/Medical Devices/Safety/AlertsandNotices/ucm473794.htm. Accessed January 13, 2019

5. U.S. Food and Drug Administration. Recognized Consensus Standards. Recognition number 3-99, AAMI TIR42:2010. Evaluation of Particulates Associated with Vascular Medical Devices. Updated September 17, 2018. https://www.accessdata.fda.gov/scripts/cdrh/cfdocs/cfStandards/ detail.cfm?standard_identification_no $=38909$. Accessed 13 January 13,2019
(D.M. Chopra

Chemical Engineer/Medical Researcher Camarillo, California

(1) J.P. Cruz

Department of Radiology Hospital Clínico de la Pontificia Universidad Católica de Chile Santiago, Chile (1) Y.C. Hu

Department of Neurosurgery University Hospitals Cleveland Medical Center Cleveland, Ohio 